\title{
Nonlinear superhorizon perturbations of non-canonical scalar field
}

\author{
Yu-ichi Takamizu囷 and Shinji Mukohyama讨 \\ 1 Department of Physics, Waseda University, Okubo 3-4-1, Shinjuku, Tokyo 169-8555, Japan \\ 2 Institute for the Physics and Mathematics of the Universe (IPMU), \\ The University of Tokyo, 5-1-5 Kashiwanoha, Kashiwa, Chiba 277-8582, Japan
}

(Dated: October 24, 2018)

\begin{abstract}
We develop a theory of non-linear cosmological perturbations at superhorizon scales for a scalar field with a Lagrangian of the form $P(X, \phi)$, where $X=-\partial^{\mu} \phi \partial_{\mu} \phi$ and $\phi$ is the scalar field. We employ the ADM formalism and the spatial gradient expansion approach to obtain general solutions valid up to the second order in the gradient expansion. This formulation can be applied to, for example, DBI inflation models to investigate superhorizon evolution of non-Gaussianities. With slight modification, we also obtain general solutions valid up to the same order for a perfect fluid with a general equation of state $P=P(\rho)$.

PACS numbers: 98.80.-k, 98.90.Cq
\end{abstract}

\section{INTRODUCTION}

Generation of primordial fluctuations during inflation is one of the most interesting predictions of quantum field theory. Indeed, those quantum fluctuations are considered as seeds of the large scale structure of the present universe, and this picture has been accepted by many researchers as a standard scenario. The cosmic microwave background (CMB) temperature anisotropy found by COBE [1] was perfectly consistent with the predictions of the linear theory of cosmological perturbations [2, 3, 4]. In particular, the primordial fluctuations are nearly scale invariant and consistent with Gaussian statistics [5].

The recent more accurate observation by WMAP, however, has revealed deviation from exact scale invariance, with a slight red tilt [6]. Moreover, there is a good possibility that deviation from Gaussianity can be detected by the future experiments such as PLANCK [7]. With those current and future precision observations, deviation from the exact scale invariance and Gaussianity can be a powerful tool to discriminate many possible inflationary models. Especially, non-Gaussianity of primordial fluctuations has recently been a focus of much attention by many authors [8, 9, 10, 11, 12, 13, 14, 15, 16, 17, 18, 19, 20, 21, 22, 23, 24, 25].

In order to parameterize the amount of non-Gaussianity of primordial perturbations, commonly used is the nonlinear parameter $f_{N L}$. This is related to the bi-spectrum of the curvature perturbation, and is defined as [16]

$$
\zeta(\mathbf{x})=\zeta_{G}(\mathbf{x})-\frac{3}{5} f_{N L} \zeta_{G}^{2}(\mathbf{x}),
$$

where $\zeta_{G}$ is the curvature perturbation on a uniform density hypersurface and satisfies linear Gaussian statistics. On the observational side, current bounds on the parameter $f_{N L}$ by WMAP five years [6] are $-9<f_{N L}<111$ for the local form of bi-spectrum and $-151<f_{N L}<253$ for the equilateral form. By future experiments such as PLANCK [7], it is expected that non-Gaussianity of the level $\left|f_{N L}\right| \gtrsim 5$ can be detected [16].

On the theoretical side, although the non-Gaussianity from the standard single-field slow-roll inflation is suppressed by slow-roll parameters [9, 15] and is too small to be detected in near-future experiments, many new types of inflationary models predicting large non-Gaussianity $\left(f_{N L}\right.$ greater than unity) have been proposed. There are at least two known mechanisms to generate large non-Gaussianity: isocurvature perturbations and non-canonical kinetic terms. Intriguingly, bi-spectrums for these two mechanism typically have different shapes: the so called local type for non-Gaussianity from isocurvature perturbations and the equilateral type for non-Gaussianity from non-canonical kinetic terms.

As for the former case, a typical example is the curvaton scenario [26, 27, 28, 29], where a light scalar field, called curvaton, is responsible for isocurvature perturbations during inflation. Another example is multi-field inflation models, where generation of isocurvature perturbations is generally expected because of the existence of more than one fields in the inflaton sector [17, 18, 19, 20]. In these examples, isocurvature perturbations generated during inflation

*Electronic address: takamizu at' gravity.phys.waseda.ac.jp

${ }^{\dagger}$ Electronic address: shinji.mukohyama at ipmu.jp 
are later converted to curvature perturbations, and this process is important for large non-Gaussianity. Now let us consider the later case, where non-canonical kinetic terms are responsible for large non-Gaussianity. Examples of this type include k-inflation [30, 31], ghost inflation [32, 33] and DBI inflation [34, 35]. In fact, Weinberg [36] has recently shown that the leading corrections to the Gaussian correlations of curvature perturbation are solely of the k-inflation type (including DBI inflation), except for ghost inflation. In k-inflation and DBI inflation, large non-Gaussianity is expected when the non-linear nature of the non-canonical kinetic action becomes significant. This happens when the sound speed of perturbations is sufficiently smaller than unity [37].

On the other hand, in ghost inflation large non-Gaussianity, such as $\left|f_{N L}\right| \simeq 80$, is always expected unless finetuned. The reason is that non-linear terms in the low energy effective Lagrangian is suppressed only by fractional (not integer) powers of energy scales. To be more precise, the leading non-linear term is suppressed only by $(H / M)^{1 / 4}$, where $H$ is the Hubble expansion rate during inflation and $M$ is the cutoff scale of the low energy effective theory. This is confirmed by a simple scaling analysis and, thus, should be robust. After using the COBE normalization $\delta \rho / \rho \simeq 10^{-5}$, which implies $H / M \simeq 10^{-4}$, this fact leads to the prediction of large non-Gaussianity [32] if the dimensionless coupling constant for the leading nonlinear term is set to be order unity, i.e. if we do not fine-tune the theory.

For these reasons, non-Gaussianity is one of the most powerful tools to distinguish models of inflation with combination of the future observations. Thus, to quantify the non-Gaussianity and clarify its observational signature, it is important to develop a theory that can deal with nonlinear cosmological perturbations. There are couple of methods to tackle this problem. One is a second-order perturbation theory [9, 38]. Another is based on spatial gradient expansion [13, 39]. While the former mainly deals with primordial perturbations up to around the horizon exit, the later deals with classical evolution after horizon exist. Thus it is important to develop both methods and to use them complementarily.

Closely related to the gradient expansion method, cosmological perturbations on superhorizon scales have been studied extensively in the so-called separate universe approach or $\delta N$ formalism 40, 41]. Actually, these approaches are essentially the leading order approximation to the gradient expansion [12, 13]. Including these, many of the previous studies were confined to the leading order approximation to the gradient expansion. However, higher order corrections to the leading order results can be important to get more detailed information about non-Gaussianity. One good example is the case studied by Leach et al [42]. They considered linear perturbations in single-field inflation models and supposed that there is a stage at which slow-roll conditions are violated. It has been then shown that, due to the decaying mode, the $O\left(\epsilon^{2}\right)$ corrections in spatial derivative expansion do affect the evolution of curvature perturbations on superhorizon scales [42]. A similar situation for DBI inflation was considered by Jain et al [43]. In these situations, it is expected that non-Gaussianities should also be affected by the order $O\left(\epsilon^{2}\right)$ effects. However, the linear perturbation theory is not capable for calculation of non-Gaussianity. Thus, it is necessary to develop nonlinear theory of cosmological perturbations valid up to $O\left(\epsilon^{2}\right)$ in the spatial gradient expansion.

Gradient expansion formalism has been developed and used by many authors [13, 24, 25, 39, 40, 41]. Formulation valid up to $O\left(\epsilon^{2}\right)$ was developed, for example, by Tanaka and Sasaki for a universe dominated by a perfect fluid with a specific equation of state $P / \rho=$ const [24] and that dominated by a canonical scalar field [25]. However, as far as the authors know, those works have not extended to a perfect fluid with general equation of state $P=P(\rho)$ nor to a scalar field with non-canonical kinetic action, which is essential for the second type of mechanism of generating non-Gaussianity.

The purpose of this paper is to fill this gap. Namely, we shall develop a theory of nonlinear superhorizon perturbations valid up to the order $O\left(\epsilon^{2}\right)$ for a scalar field with non-canonical kinetic action and a perfect fluid with general equation of state.

This paper is organized as follows. In Sec. III, we introduce a non-canonical scalar field as our model and express it in a perfect fluid form. In Sec. III, we shall develop a theory of nonlinear cosmological perturbations on superhorizon scales and explain our formulation in details. The following Sec. IV is devoted to some details of obtaining a general solution. We then study some specific examples in Sec. V V Sec. VI is devoted to a summary of this paper and discussion. In Appendix, we give our result in a perfect fluid system.

\section{SCALAR FIELD IN A PERFECT FLUID FORM}

Throughout this paper we consider a minimally-coupled scalar field described by an action of the form

$$
I=\int d^{4} x \sqrt{-g} P(X, \phi)
$$


where $X=-g^{\mu \nu} \partial_{\mu} \phi \partial_{\nu} \phi$, and suppose that $-g^{\mu \nu} \partial_{\nu} \phi$ is timelike and future-directed. The equation of motion for $\phi$ is

$$
\frac{2}{\sqrt{-g}} \partial_{\mu}\left(\sqrt{-g} P_{X} \partial^{\mu} \phi\right)+P_{\phi}=0
$$

where the subscripts $X$ and $\phi$ represent derivative with respect to $X$ and $\phi$, respectively. The stress energy tensor of the scalar field is shown to be a perfect fluid form:

$$
T_{\mu \nu}=2 P_{X} \partial_{\mu} \phi \partial_{\nu} \phi+P g_{\mu \nu}=(\rho+P) u_{\mu} u_{\nu}+P g_{\mu \nu}
$$

where

$$
\rho(X, \phi)=2 P_{X} X-P, \quad u_{\mu}=-\frac{\partial_{\mu} \phi}{\sqrt{X}}
$$

Note that $u^{\mu} u_{\mu}=-1$. As far as $\partial_{\mu} \phi \neq 0$, the equation of motion (2.2) is equivalent to the conservation equation $\nabla_{\mu} T_{\nu}^{\mu}=0$.

The following relation among first-order variations of $P, \rho$ and $\phi$ will be useful in the analysis below.

$$
\delta P=c_{s}^{2} \delta \rho+\rho \Gamma \delta \phi
$$

where

$$
c_{s}^{2}=\frac{P_{X}}{2 P_{X X} X+P_{X}}, \quad \Gamma=\frac{1}{\rho}\left(P_{\phi}-c_{s}^{2} \rho_{\phi}\right) .
$$

Note that $c_{s}$ is the speed of sound for the gauge invariant scalar perturbation in the linear theory [30].

\section{FORMALISM}

In this section we shall develop a theory of nonlinear cosmological perturbations on superhorizon scales. For this purpose we employ the ADM formalism and the gradient expansion in the uniform Hubble slicing.

\section{A. ADM decomposition}

In the $(3+1)$-decomposition, the metric is expressed as

$$
d s^{2}=g_{\mu \nu} d x^{\mu} d x^{\nu}=-\alpha^{2} d t^{2}+\gamma_{i j}\left(d x^{i}+\beta^{i} d t\right)\left(d x^{j}+\beta^{j} d t\right)
$$

where $\alpha$ is the lapse function, $\beta^{i}$ is the shift vector and Latin indices run over $1,2,3$. Since $\alpha$ and $\beta^{i}$ represent gauge degrees of freedom for diffeomorphism and appear as Lagrange multipliers in the action, the corresponding equations of motion leads to constraint equations. Contrary to $\alpha$ and $\beta$, components of the spatial metric $\gamma_{i j}$ are dynamical variables (subject to the constraint equations) and the corresponding equations of motion are called dynamical equations. In what follows we shall express the dynamical equations as a set of first-order differential equations with respect to the time $t$. For this purpose we introduce the extrinsic curvature $K_{i j}$ defined by

$$
K_{i j}=-\frac{1}{2 \alpha}\left(\partial_{t} \gamma_{i j}-D_{i} \beta_{j}-D_{j} \beta_{i}\right)
$$

where $D$ is the covariant derivative compatible with the spatial metric $\gamma_{i j}$. For the stress-energy tensor in the perfect fluid form (2.3), we define the 3 -vector $v^{i}$ as $v^{i} \equiv u^{i} / u^{0}$. Hereafter, we shall use $\gamma_{i j}$ and its inverse $\gamma^{i j}$ to raise and lower indices of $K, D, v, \beta$. Then we can express $u^{\mu}$ and $u_{\mu}$ in terms of $\alpha, \beta^{i}$ and $v^{i}$ :

$$
\begin{aligned}
u^{0} & =\left[\alpha^{2}-\left(v_{k}+\beta_{k}\right)\left(v^{k}+\beta^{k}\right)\right]^{-1 / 2}, \\
u^{i} & =u^{0} v^{i} \\
u_{0} & =-u^{0}\left[\alpha^{2}-\beta_{k}\left(v^{k}+\beta^{k}\right)\right] \\
u_{i} & =u^{0}\left(v_{i}+\beta_{i}\right)
\end{aligned}
$$


The conservation equation $\nabla_{\mu} T_{\nu}^{\mu}=0$ is

$$
\begin{aligned}
& u^{\mu} \partial_{\mu} \rho+\frac{\rho+P}{\alpha \sqrt{\operatorname{det} \gamma}} \partial_{\mu}\left(\alpha \sqrt{\operatorname{det} \gamma} u^{\mu}\right)=0, \\
& \frac{1}{\sqrt{\operatorname{det} \gamma}} \partial_{t}\left[\sqrt{\operatorname{det} \gamma}(\rho+P) w u_{i}\right]+D_{j}\left[(\rho+P) w v^{j} u_{i}\right]=-\alpha \partial_{i} P-(\rho+P) w\left[w \partial_{i} \alpha-u_{j} D_{i} \beta^{j}\right] \text {, }
\end{aligned}
$$

where $w \equiv \alpha u^{0}$. All independent components of the stress energy tensor are conveniently expressed as

$$
\begin{aligned}
E & \equiv T_{\mu \nu} n^{\mu} n^{\nu}=(\rho+P) w^{2}-P, \\
J_{i} & \equiv-T_{\mu i} n^{\mu}=(\rho+P) w u_{i}, \\
S_{i j} & \equiv T_{i j}=(\rho+P) u_{i} u_{j}+P \gamma_{i j},
\end{aligned}
$$

where $n^{\mu}$ is the unit vector normal to the constant $t$ surfaces and is given by

$$
n_{\mu} d x^{\mu}=-\alpha d t, \quad n^{\mu} \partial_{\mu}=\frac{1}{\alpha}\left(\partial_{t}-\beta^{i} \partial_{i}\right)
$$

The Hamiltonian constraint, corresponding to the equation of motion for $\alpha$, is

$$
R+K^{2}-K_{i j} K^{i j}=2 \kappa^{2} E
$$

where $\kappa^{2}=8 \pi G_{N}, R \equiv R[\gamma]$ is the Ricci scalar of the spatial metric $\gamma$ and $K \equiv K_{i}^{i}$. The momentum constraint, corresponding to the equation of motion for $\beta^{i}$, is

$$
-\partial_{i} K+D_{j} K_{i}^{j}=\kappa^{2} J_{i}
$$

The dynamical equations, decomposed into the trace part and the traceless part, are

$$
\begin{aligned}
\partial_{\perp} K-K_{i j} K^{i j}+\frac{D^{2} \alpha}{\alpha} & =\frac{\kappa^{2}}{2}\left(S_{k}^{k}+E\right), \\
R_{i j}-\nabla_{\perp} K_{i j}+K K_{i j}-\frac{D_{i} D_{j} \alpha}{\alpha}-\frac{\gamma_{i j}}{3}\left[R-\partial_{\perp} K+K^{2}-\frac{D^{2} \alpha}{\alpha}\right] & =\kappa^{2}\left(S_{i j}-\frac{1}{3} S_{k}^{k} \gamma_{i j}\right),
\end{aligned}
$$

where $\partial_{\perp} \equiv n^{\mu} \partial_{\mu}, \nabla_{\perp} \equiv n^{\mu} \nabla_{\mu}, D^{2} \equiv \gamma^{i j} D_{i} D_{j}$ and $R_{i j} \equiv R_{i j}[\gamma]$ is the Ricci tensor of the spatial metric $\gamma$.

In addition to the standard ADM decomposition briefly reviewed above, we further decompose the spatial metric and the extrinsic curvature as

$$
\begin{aligned}
\gamma_{i j} & =a^{2} \psi^{4} \tilde{\gamma}_{i j}, \\
K_{i j} & =a^{2} \psi^{4}\left(\frac{1}{3} K \tilde{\gamma}_{i j}+\tilde{A}_{i j}\right),
\end{aligned}
$$

where $a(t)$ is the scale factor of a fiducial Friedmann background (specified later) and the determinant of $\tilde{\gamma}_{i j}$ is constrained to be unity: $\operatorname{det} \tilde{\gamma}_{i j}=1$. The first-order equations for the spatial metric $\left(\psi, \tilde{\gamma}_{i j}\right)$ are deduced from the definition of the extrinsic curvature (3.2) as

$$
\begin{aligned}
\frac{\partial_{\perp} \psi}{\psi}+\frac{\partial_{t} a}{2 \alpha a} & =\frac{1}{6}\left(-K+\frac{\partial_{i} \beta^{i}}{\alpha}\right) \\
\partial_{\perp} \tilde{\gamma}_{i j} & =-2 \tilde{A}_{i j}+\frac{1}{\alpha}\left(\tilde{\gamma}_{i k} \partial_{j} \beta^{k}+\tilde{\gamma}_{j k} \partial_{i} \beta^{k}-\frac{2}{3} \tilde{\gamma}_{i j} \partial_{k} \beta^{k}\right) .
\end{aligned}
$$

The first-order equations for the extrinsic curvature $\left(K, \tilde{A}_{i j}\right)$ are obtained from the dynamical equations (3.9) as

$$
\begin{aligned}
\partial_{\perp} K= & \frac{K^{2}}{3}+\tilde{A}^{i j} \tilde{A}_{i j}-\frac{D^{2} \alpha}{\alpha}+\frac{\kappa^{2}}{2}\left(S_{k}^{k}+E\right) \\
\partial_{\perp} \tilde{A}_{i j}= & \left(K \tilde{A}_{i j}-2 \tilde{A}_{i}{ }^{k} \tilde{A}_{k j}\right)+\frac{1}{\alpha}\left(\tilde{A}_{i k} \partial_{j} \beta^{k}+\tilde{A}_{j k} \partial_{i} \beta^{k}-\frac{2}{3} \tilde{A}_{i j} \partial_{k} \beta^{k}\right) \\
& +\frac{1}{a^{2} \psi^{4}}\left[\left(R_{i j}-\frac{R}{3} \gamma_{i j}\right)-\frac{1}{\alpha}\left(D_{i} D_{j} \alpha-\frac{D^{2} \alpha}{3} \gamma_{i j}\right)-\kappa^{2}\left(S_{i j}-\frac{S_{k}^{k}}{3} \gamma_{i j}\right)\right],
\end{aligned}
$$


where $\tilde{A}_{i}{ }^{k}=\tilde{\gamma}^{j k} \tilde{A}_{i j}$ and $\tilde{A}^{j k}=\tilde{\gamma}^{i j} \tilde{A}_{i}{ }^{k}$. The Hamiltonian and momentum constraints are, respectively,

$$
\begin{aligned}
R+\frac{2}{3} K^{2}-\tilde{A}^{i j} \tilde{A}_{i j} & =2 \kappa^{2} E \\
-\frac{2}{3} \partial_{i} K+D_{j} \tilde{A}_{i}^{j} & =\kappa^{2} J_{i} .
\end{aligned}
$$

The conservation equation is

$$
\begin{array}{r}
\left(\partial_{t}+v^{i} \partial_{i}\right) \rho+\frac{\rho+P}{\left(a \psi^{2}\right)^{3} w}\left\{\partial_{t}\left[\left(a \psi^{2}\right)^{3} w\right]+\partial_{i}\left[\left(a \psi^{2}\right)^{3} w v^{i}\right]\right\}=0, \\
\frac{1}{\left(a \psi^{2}\right)^{3}} \partial_{t}\left[\left(a \psi^{2}\right)^{3}(\rho+P) w u_{i}\right]+D_{j}\left[(\rho+P) w v^{j} u_{i}\right]+\partial_{i} P+(\rho+P)\left(w^{2} \partial_{i} \alpha-w u_{j} D_{i} \beta^{j}\right)=0 .
\end{array}
$$

Throughout this paper we adopt the uniform Hubble slicing

$$
K=-3 H(t), \quad H(t) \equiv \frac{\partial_{t} a}{a} .
$$

Substituted into (3.11), this implies that

$$
\chi(\equiv \alpha-1)=\frac{2 \partial_{t} \psi}{H \psi}-\frac{D_{i} \beta^{i}}{3 H}
$$

\section{B. Gradient expansion: basic assumptions and order estimates}

In the gradient expansion approach we introduce a flat FRW universe $\left(a(t), \phi_{0}(t)\right)$ as a background and suppose that the characteristic length scale $L$ of perturbations is longer than the Hubble length scale $1 / H$ of the background, i.e. $H L \gg 1$. Therefore, we consider $\epsilon \equiv 1 /(H L)$ as a small parameter and systematically expand our equations by $\epsilon$, considering a spatial derivative acted on perturbations is of order $O(\epsilon)$.

The background flat FRW universe $\left(a(t), \phi_{0}(t)\right)$ satisfies the Friedmann equation and the equation of motion

$$
H^{2}=\frac{\kappa^{2}}{3} \rho_{0}, \quad \frac{2}{a^{3}} \partial_{t}\left(a^{3} P_{0 X} \partial_{t} \phi_{0}\right)-P_{0 \phi}=0,
$$

where $\rho_{0} \equiv \rho\left(X_{0}, \phi_{0}\right), P_{0 X} \equiv P_{X}\left(X_{0}, \phi_{0}\right), P_{0 \phi} \equiv P_{\phi}\left(X_{0}, \phi_{0}\right)$, and $X_{0} \equiv\left(\partial_{t} \phi_{0}\right)^{2}$.

Since the FRW background is recovered in the limit $\epsilon \rightarrow 0$, we naturally have the estimates

$$
v^{i}=O(\epsilon), \quad \beta^{i}=O(\epsilon),
$$

and $\partial_{t} \tilde{\gamma}_{i j}=O(\epsilon)$. Actually, following the arguments in refs. [24, 25], we assume a stronger condition

$$
\partial_{t} \tilde{\gamma}_{i j}=O\left(\epsilon^{2}\right) .
$$

This assumption significantly simplifies our analysis and, we believe, still allows many useful applications of the formalism. On the other hand, we consider $\psi$ and $\tilde{\gamma}_{i j}$ (without derivatives acted on them) as quantities of order $O(1)$.

We can estimate orders of magnitude of various quantities by using the above assumption and the basic equations. First, (3.12) implies that

$$
\tilde{A}_{i j}=O\left(\epsilon^{2}\right) .
$$

Substituting (3.24) into (3.16) we obtain $J_{i}=O\left(\epsilon^{3}\right)$, or

$$
v_{i}+\beta_{i}=O\left(\epsilon^{3}\right)
$$

For the scalar field system, this is expressed as $\partial_{i} \pi=O\left(\epsilon^{3}\right)$, where $\pi \equiv \phi-\phi_{0}$. By absorbing a homogeneous part of $\pi$ into $\phi_{0}$ (and modifying $a(t)$ accordingly), we obtain

$$
\pi=O\left(\epsilon^{2}\right)
$$


Combining (3.25) with the first equation in (3.3), we obtain $u^{0}=1 / \alpha+O\left(\epsilon^{6}\right)$, or

$$
w\left(\equiv \alpha u^{0}\right)=1+O\left(\epsilon^{6}\right) .
$$

This implies that $E=\rho+O\left(\epsilon^{6}\right)$. Thus, from (3.15) we obtain

$$
\delta\left(\equiv \frac{\rho-\rho_{0}}{\rho_{0}}\right)=O\left(\epsilon^{2}\right)
$$

and (2.5) implies that

$$
p\left(\equiv P-P_{0}\right)=O\left(\epsilon^{2}\right) .
$$

Finally, (3.17) implies that

$$
\partial_{t} \psi=O\left(\epsilon^{2}\right)
$$

and thus we obtain

$$
\chi=O\left(\epsilon^{2}\right)
$$

from the uniform Hubble slicing condition (3.20).

In summary, we have the following estimates (including assumptions):

$$
\begin{aligned}
& \psi=O(1), \quad \tilde{\gamma}_{i j}=O(1), \quad v^{i}=O(\epsilon), \quad \beta^{i}=O(\epsilon), \\
& \chi=O\left(\epsilon^{2}\right), \quad \tilde{A}_{i j}=O\left(\epsilon^{2}\right), \quad \delta=O\left(\epsilon^{2}\right), \quad \pi=O\left(\epsilon^{2}\right), \quad p=O\left(\epsilon^{2}\right), \\
& \partial_{t} \tilde{\gamma}_{i j}=O\left(\epsilon^{2}\right), \quad \partial_{t} \psi=O\left(\epsilon^{2}\right), \quad v^{i}+\beta^{i}=O\left(\epsilon^{3}\right), \quad w=1+O\left(\epsilon^{6}\right) .
\end{aligned}
$$

\section{Leading order equations}

Substituting the order of magnitude shown in (3.32) into the conservation equations (3.17) and (3.18), we find

$$
\begin{aligned}
\rho_{0} \partial_{t} \delta+\left(\rho_{0}+P_{0}\right)\left(6 \frac{\partial_{t} \psi}{\psi}+D_{i} v^{i}\right)+3 H\left(p-P_{0} \delta\right) & =O\left(\epsilon^{4}\right), \\
\frac{1}{a^{3}} \partial_{t}\left[a^{3}\left(\rho_{0}+P_{0}\right) u_{i}\right]+\partial_{i}\left[p+\left(\rho_{0}+P_{0}\right) \chi\right] & =O\left(\epsilon^{5}\right) .
\end{aligned}
$$

The Hamiltonian and momentum constraint equations give

$$
\begin{aligned}
8 \frac{\tilde{D}^{2} \psi}{\psi} & =\tilde{R}-2 \kappa^{2} a^{2} \psi^{4} \rho_{0} \delta+O\left(\epsilon^{4}\right), \\
\tilde{D}_{j}\left(\psi^{6} \tilde{A}_{i}^{j}\right) & =\kappa^{2}\left(\rho_{0}+P_{0}\right) \psi^{6} u_{i}+O\left(\epsilon^{5}\right),
\end{aligned}
$$

where $\tilde{R} \equiv R[\tilde{\gamma}]$ is the Ricci scalar of the normalized spatial metric $\tilde{\gamma}_{i j}, \tilde{D}$ is the covariant derivative compatible with $\tilde{\gamma}_{i j}, \tilde{D}^{2} \equiv \tilde{\gamma}^{i j} \tilde{D}_{i} \tilde{D}_{j}$, and $\tilde{\gamma}^{i j}$ is the inverse matrix of $\tilde{\gamma}_{i j}$. The evolution equations for the spatial metric give

$$
\begin{aligned}
6 \frac{\partial_{t} \psi}{\psi} & =3 H \chi+D_{i} \beta^{i}+O\left(\epsilon^{4}\right), \\
\left(\partial_{t}-\beta^{k} \partial_{k}\right) \tilde{\gamma}_{i j} & =-2 \tilde{A}_{i j}+\tilde{\gamma}_{i k} \partial_{j} \beta^{k}+\tilde{\gamma}_{j k} \partial_{i} \beta^{k}-\frac{2}{3} \tilde{\gamma}_{i j} \partial_{k} \beta^{k}+O\left(\epsilon^{4}\right),
\end{aligned}
$$

while the evolution equations for the extrinsic curvature give

$$
\begin{aligned}
& \partial_{t} \tilde{A}_{i j}+3 H \tilde{A}_{i j}=\frac{1}{a^{2} \psi^{4}}\left(R_{i j}-\frac{1}{3} R \gamma_{i j}\right)+O\left(\epsilon^{4}\right), \\
& p+\left(\rho_{0}+P_{0}\right) \chi=-\frac{1}{3} \rho_{0} \delta+O\left(\epsilon^{4}\right),
\end{aligned}
$$


By using (3.37), (3.40) and the background conservation equation $\partial_{t} \rho_{0}+3 H\left(\rho_{0}+P_{0}\right)=0$, a single equation for $\delta$ is easily obtained from (3.33),

$$
\partial_{t}\left(a^{2} \rho_{0} \delta\right)=O\left(\epsilon^{4}\right)
$$

Using (3.40) again, (3.34) is simplified to

$$
\frac{1}{a^{3}} \partial_{t}\left[a^{3}\left(\rho_{0}+P_{0}\right) u_{i}\right]=\frac{1}{3} \rho_{0} \partial_{i} \delta+O\left(\epsilon^{5}\right) .
$$

It is intriguing to note that we have not yet specified the form of $p$. Therefore, eqs. (3.33)-(3.42) hold as far as the stress-energy tensor is of the perfect fluid form and $p=O\left(\epsilon^{2}\right)$, regardless of whether the stress-energy tensor is provided by a scalar field, radiation, dust, or any other sources.

The form of $p$ for the scalar field system is specified by the relation (2.5) as

$$
p=\rho_{0}\left(c_{s 0}^{2} \delta+\Gamma_{0} \pi\right)+O\left(\epsilon^{4}\right),
$$

where $c_{s 0}^{2}=P_{0 X} /\left(2 P_{0 X X} X_{0}+P_{0 X}\right)$ and $\Gamma_{0}=\left(P_{0 \phi}-c_{s 0}^{2} \rho_{0 \phi}\right) / \rho_{0}$. We can obtain another equation relating $p$ and $\pi$, by expanding $p$ as $p=P_{0 X}\left(X-X_{0}\right)+P_{0 \phi} \pi+O\left(\epsilon^{4}\right)$, where $X-X_{0}=2\left(\partial_{t} \phi_{0} \partial_{t} \pi-\chi X_{0}\right)+O\left(\epsilon^{4}\right)$. Actually, this equation can be interpreted as a first-order equation for $\pi$. Using (3.40), $\rho_{0}+P_{0}=2 P_{0 X} X_{0}$ and the background equation of motion (3.21), we can rewrite this equation for $\pi$ as

$$
\frac{1}{a^{3}} \partial_{t}\left[\frac{\left(\rho_{0}+P_{0}\right) a^{3}}{\partial_{t} \phi_{0}} \pi\right]=-\frac{1}{3} \rho_{0} \delta+O\left(\epsilon^{4}\right) .
$$

\section{GENERAL SOLUTION}

Having written down all relevant equations up to the order $O\left(\epsilon^{2}\right)$ in the gradient expansion, we now seek a general solution.

\section{A. Leading order solutions}

First, $\psi=O(1)$ and $\partial_{t} \psi=O\left(\epsilon^{2}\right)$ imply that

$$
\psi=L^{(0)}\left(x^{k}\right)+O\left(\epsilon^{2}\right),
$$

where $L^{(0)}\left(x^{k}\right)$ is an arbitrary function of the spatial coordinates $\left\{x^{k}\right\}(k=1,2,3)$. Hereafter, the superscript $(n)$ indicates that the corresponding quantity is of order $O\left(\epsilon^{n}\right)$. Similarly, $\tilde{\gamma}_{i j}=O(1)$ and $\partial_{t} \tilde{\gamma}_{i j}=O\left(\epsilon^{2}\right)$ imply that

$$
\tilde{\gamma}_{i j}=f_{i j}^{(0)}\left(x^{k}\right)+O\left(\epsilon^{2}\right),
$$

where $f_{i j}^{(0)}\left(x^{k}\right)$ is a $(3 \times 3)$-matrix with unit determinant whose components depend only on the spatial coordinates. With these expressions, the right hand side of (3.39) is calculated as

$$
\frac{1}{a^{2} \psi^{4}}\left(R_{i j}-\frac{1}{3} R \gamma_{i j}\right)=\frac{1}{a^{2}} F_{i j}^{(2)}\left(x^{k}\right)+O\left(\epsilon^{4}\right),
$$

where

$$
\begin{aligned}
F_{i j}^{(2)}\left(x^{k}\right) \equiv & \frac{1}{\left(L^{(0)}\right)^{4}}\left[\left(\tilde{R}_{i j}^{(0)}-\frac{1}{3} \tilde{R}^{(0)} f_{i j}^{(0)}\right)+2\left(2 \partial_{i} \ln L^{(0)} \partial_{j} \ln L^{(0)}-\tilde{D}_{i}^{(0)} \tilde{D}_{j}^{(0)} \ln L^{(0)}\right)\right. \\
& \left.-\frac{2}{3} f_{(0)}^{k l}\left(2 \partial_{k} \ln L^{(0)} \partial_{l} \ln L^{(0)}-\tilde{D}_{k}^{(0)} \tilde{D}_{l}^{(0)} \ln L^{(0)}\right) f_{i j}^{(0)}\right],
\end{aligned}
$$

$f_{(0)}^{k l}$ is the inverse matrix of $f_{i j}^{(0)}, \tilde{R}_{i j}^{(0)}=R_{i j}\left[f^{(0)}\right]$ and $\tilde{R}^{(0)}=R\left[f^{(0)}\right]$ are Ricci tensor and Ricci scalar of the 0th-order spatial metric $f_{i j}^{(0)}$, and $\tilde{D}^{(0)}$ is the covariant derivative compatible with $f_{i j}^{(0)}$. Note that $f_{(0)}^{i j} F_{i j}^{(2)}=0$ by definition. Thus, we obtain

$$
\tilde{A}_{i j}=\frac{1}{a^{3}(t)}\left[F_{i j}^{(2)}\left(x^{k}\right) \int_{t_{0}}^{t} a\left(t^{\prime}\right) d t^{\prime}+C_{i j}^{(2)}\left(x^{k}\right)\right]+O\left(\epsilon^{4}\right)
$$


where $C_{i j}^{(2)}\left(x^{k}\right)$ is a symmetric matrix whose components depend only on the spatial coordinates and which satisfies $f_{(0)}^{i j} C_{i j}^{(2)}=0$.

Next, the equation (3.41) for $\delta$ is easily solved as

$$
\delta=\frac{\rho_{*} a_{*}^{2}}{\rho_{0}(t) a^{2}(t)} Q^{(2)}\left(x^{k}\right)+O\left(\epsilon^{4}\right)
$$

where $\rho_{*} a_{*}^{2}$ is a constant and $Q^{(2)}\left(x^{k}\right)$ is an arbitrary function of the spatial coordinates. With this expression for $\delta$, (3.42) gives

$$
u_{i}=\frac{\rho_{*} a_{*}^{2}}{3\left[\rho_{0}(t)+P_{0}(t)\right] a^{3}(t)}\left[\partial_{i} Q^{(2)}\left(x^{k}\right) \int_{t_{0}}^{t} a\left(t^{\prime}\right) d t^{\prime}+C_{i}^{(3)}\left(x^{k}\right)\right]+O\left(\epsilon^{5}\right)
$$

where $C_{i}^{(3)}\left(x^{k}\right)$ is an arbitrary function of the spatial coordinates.

The 'constants' of integration $L^{(0)}\left(x^{k}\right), f_{i j}^{(0)}\left(x^{k}\right), C_{i j}^{(2)}\left(x^{k}\right), Q^{(2)}\left(x^{k}\right)$ and $C_{i}^{(3)}\left(x^{k}\right)$ are not independent. Indeed, by solving the Hamiltonian and momentum constraints (3.35) and (3.36), $Q^{(2)}$ and $C_{i}^{(3)}$ are expressed in terms of other integration 'constants' as

$$
\begin{aligned}
Q^{(2)} & =\frac{1}{2 \kappa^{2} \rho_{*} a_{*}^{2}} R\left[\left(L^{(0)}\right)^{4} f^{(0)}\right]+O\left(\epsilon^{4}\right), \\
C_{i}^{(3)} & =\frac{3}{\left(L^{(0)}\right)^{6} \kappa^{2} \rho_{*} a_{*}^{2}} f_{(0)}^{j k} \tilde{D}_{j}^{(0)}\left[\left(L^{(0)}\right)^{6} C_{k i}^{(2)}\right]+O\left(\epsilon^{5}\right) .
\end{aligned}
$$

Until now, we have not used either (3.43) or (3.44). Therefore, the general solutions presented above are valid not only for the scalar field system but also for radiation, dust or any other sources, provided that the stress-energy tensor is of the perfect fluid form and that $p=O\left(\epsilon^{2}\right)$.

We now use (3.43) and (3.44) to proceed further. It is easy to integrate (3.44) to give

$$
\pi=-\frac{\rho_{*} a_{*}^{2} \partial_{t} \phi_{0}}{3\left(\rho_{0}+P_{0}\right) a^{3}}\left[Q^{(2)}\left(x^{k}\right) \int_{t_{0}}^{t} a\left(t^{\prime}\right) d t^{\prime}+\Pi^{(2)}\left(x^{k}\right)\right]+O\left(\epsilon^{4}\right),
$$

where $\Pi^{(2)}\left(x^{k}\right)$ is an arbitrary function of the spatial coordinates. By using (3.43) and (3.40) we obtain

$$
\chi=-\frac{\rho_{*} a_{*}^{2}}{3\left(\rho_{0}+P_{0}\right) a^{2}}\left[\left(1+3 c_{s 0}^{2}-\frac{\rho_{0} \Gamma_{0} \partial_{t} \phi_{0}}{\left(\rho_{0}+P_{0}\right) a} \int_{t_{0}}^{t} a\left(t^{\prime}\right) d t^{\prime}\right) Q^{(2)}\left(x^{k}\right)-\frac{\rho_{0} \Gamma_{0} \partial_{t} \phi_{0}}{\left(\rho_{0}+P_{0}\right) a} \Pi^{(2)}\left(x^{k}\right)\right]+O\left(\epsilon^{4}\right) .
$$

Note that, since $u_{i}=-\partial_{i} \phi / \sqrt{X}=-\partial_{i} \pi / \partial_{t} \phi_{0}+O\left(\epsilon^{5}\right)$, the integration 'constant' $\Pi^{(2)}\left(x^{k}\right)$ is related to $C_{i}^{(3)}\left(x^{k}\right)$ as

$$
C_{i}^{(3)}=\partial_{i} \Pi^{(2)}+O\left(\epsilon^{5}\right)
$$

Thus, $C_{i}^{(3)}$ for the scalar field system does not include a transverse part.

\section{B. Solution up to $O\left(\epsilon^{2}\right)$}

Solutions obtained so far are correct up to leading order in the gradient expansion. Among them, the spatial metric $\psi$ and $\tilde{\gamma}_{i j}$ have been obtained only up to $O(1)$ while all other variables are correct at least up to $O\left(\epsilon^{2}\right)$. In this subsection we seek $O\left(\epsilon^{2}\right)$ corrections to $\psi$ and $\tilde{\gamma}_{i j}$. For this purpose it is convenient to specify the shift vector $\beta^{i}$ more accurately than indicated by (3.32): in this subsection we set

$$
\beta=O\left(\epsilon^{3}\right)
$$

With this gauge choice, 3.38 is reduced to

$$
\partial_{t} \tilde{\gamma}_{i j}=-2 \tilde{A}_{i j}+O\left(\epsilon^{4}\right),
$$


and thus results in

$$
\tilde{\gamma}_{i j}=f_{i j}^{(0)}\left(x^{k}\right)-2\left[F_{i j}^{(2)}\left(x^{k}\right) \int_{t_{0}}^{t} \frac{d t^{\prime}}{a^{3}\left(t^{\prime}\right)} \int_{t_{0}}^{t^{\prime}} a\left(t^{\prime \prime}\right) d t^{\prime \prime}+C_{i j}^{(2)}\left(x^{k}\right) \int_{t_{0}}^{t} \frac{d t^{\prime}}{a^{3}\left(t^{\prime}\right)}\right]+O\left(\epsilon^{4}\right),
$$

where we have absorbed a new integration 'constant' into the 0 -th order integration 'constant' $f_{i j}^{(0)}\left(x^{k}\right)$. Again, we have not used either (3.43) or (3.44) to derive this result. Therefore, the general solution (4.14) is valid not only for the scalar field system but also for any other sources, provided that the stress-energy tensor is of the perfect fluid form, that $p=O\left(\epsilon^{2}\right)$ and that the additional gauge condition (4.12) is imposed.

In order to seek the $O\left(\epsilon^{2}\right)$ correction to $\psi$, we specialize to the scalar field system since we will need the solution (4.10) for $\chi$, which was obtained by using (3.43). The evolution equation (3.37) with (4.1) leads to

$$
\ln \left[\frac{\psi}{L^{(0)}\left(x^{k}\right)}\right]=\frac{1}{2} \int_{t_{0}}^{t} H\left(t^{\prime}\right) \chi\left(t^{\prime}\right) d t^{\prime}+O\left(\epsilon^{4}\right)
$$

where we have absorbed a new integration 'constant' into the 0-th order integration 'constant' $L^{(0)}\left(x^{k}\right)$. We can substitute (4.10) to the right hand side of this equation to complete the procedure.

\section{Summary of the solution}

In summary we have obtained the following solutions in the gradient expansion for the scalar field system.

$$
\begin{aligned}
\delta= & \frac{1}{2 \kappa^{2} \rho_{0} a^{2}} R\left[\left(L^{(0)}\right)^{4} f^{(0)}\right]+O\left(\epsilon^{4}\right), \\
u_{i}= & \frac{1}{6 \kappa^{2}\left(\rho_{0}+P_{0}\right) a^{3}} \partial_{i}\left(R\left[\left(L^{(0)}\right)^{4} f^{(0)}\right] \int_{t_{0}}^{t} a\left(t^{\prime}\right) d t^{\prime}+C^{(2)}\right)+O\left(\epsilon^{5}\right), \\
\pi= & -\frac{\partial_{t} \phi_{0}}{6 \kappa^{2}\left(\rho_{0}+P_{0}\right) a^{3}}\left(R\left[\left(L^{(0)}\right)^{4} f^{(0)}\right] \int_{t_{0}}^{t} a\left(t^{\prime}\right) d t^{\prime}+C^{(2)}\right)+O\left(\epsilon^{4}\right), \\
\chi= & -\frac{1}{6 \kappa^{2}\left(\rho_{0}+P_{0}\right) a^{2}}\left[\left(1+3 c_{s 0}^{2}-\frac{\rho_{0} \Gamma_{0} \partial_{t} \phi_{0}}{\left(\rho_{0}+P_{0}\right) a} \int_{t_{0}}^{t} a\left(t^{\prime}\right) d t^{\prime}\right) R\left[\left(L^{(0)}\right)^{4} f^{(0)}\right]-\frac{\rho_{0} \Gamma_{0} \partial_{t} \phi_{0}}{\left(\rho_{0}+P_{0}\right) a} C^{(2)}\right] \\
& +O\left(\epsilon^{4}\right), \\
\psi= & L^{(0)}\left(1+\frac{1}{2} \int_{t_{0}}^{t} H\left(t^{\prime}\right) \chi\left(t^{\prime}\right) d t^{\prime}\right)+O\left(\epsilon^{4}\right), \\
\tilde{\gamma}_{i j}= & f_{i j}^{(0)}-2\left(F_{i j}^{(2)} \int_{t_{0}}^{t} \frac{d t^{\prime}}{a^{3}\left(t^{\prime}\right)} \int_{t_{0}}^{t^{\prime}} a\left(t^{\prime \prime}\right) d t^{\prime \prime}+C_{i j}^{(2)} \int_{t_{0}}^{t} \frac{d t^{\prime}}{a^{3}\left(t^{\prime}\right)}\right)+O\left(\epsilon^{4}\right), \\
\tilde{A}_{i j}= & \frac{1}{a^{3}}\left(F_{i j}^{(2)} \int_{t_{0}}^{t} a\left(t^{\prime}\right) d t^{\prime}+C_{i j}^{(2)}\right)+O\left(\epsilon^{4}\right),
\end{aligned}
$$

where $C^{(2)}$ in this expression is related to $\Pi^{(2)}$ in (4.10) as $C^{(2)}=2 \kappa^{2} \rho_{*} a_{*}^{2} \Pi^{(2)}$, and $F_{i j}^{(2)}$ is defined by (4.4), and 'constants' of integration $L^{(0)}, f_{i j}^{(0)}, C^{(2)}$ and $C_{i j}^{(2)}$ depend only on the spatial coordinates $\left\{x^{k}\right\}(k=1,2,3)$, and satisfy

$$
\begin{aligned}
f_{i j}^{(0)} & =f_{j i}^{(0)}, \quad \operatorname{det}\left(f_{i j}^{(0)}\right)=1, \\
C_{i j}^{(2)} & =C_{j i}^{(2)}, \quad f_{(0)}^{i j} C_{i j}^{(2)}=0, \\
\left(L^{(0)}\right)^{6} \partial_{i} C^{(2)} & =6 f_{(0)}^{j k} \tilde{D}_{j}^{(0)}\left[\left(L^{(0)}\right)^{6} C_{k i}^{(2)}\right] .
\end{aligned}
$$

Here, $f_{(0)}^{i j}$ is the inverse matrix of $f_{i j}^{(0)}$ and $\tilde{D}^{(0)}$ is the covariant derivative compatible with $f_{i j}^{(0)}$.

Note that the gauge condition (4.12) is unchanged under purely spatial coordinate transformations

$$
x^{i} \rightarrow x^{i}=f^{i}\left(x^{k}\right) .
$$


Thus, the 0-th order spatial metric $f_{i j}^{(0)}$ includes 3 gauge degrees of freedom. Therefore, the number of degrees of freedom included in each 'constant' of integration is

$$
\begin{array}{llll}
L^{(0)} & \cdots & 1 \text { scalar growing mode }=1 \text { component } \\
f_{i j}^{(0)} & \cdots & 2 \text { tensor growing modes }=5 \text { components }-3 \text { gauge } \\
C^{(2)} & \cdots & 1 \text { scalar decaying mode }=1 \text { component, } \\
C_{i j}^{(2)} & \cdots & 2 \text { tensor decaying modes }=5 \text { components }-3 \text { constraints } .
\end{array}
$$

\section{EXAMPLES}

Until now, we have not specified a form of the function $P(X, \phi)$. In this section, we shall consider some specific examples.

\section{A. Scalar with shift symmetry}

Let us consider the case where the Lagrangian $P$ depends on the scalar field only through $X$, i.e. $P=P(X)$. This case corresponds to a scalar with shift symmetry and is often considered in models of k-inflation [31].

The sound speed $c_{s 0}^{2}$ and $\Gamma_{0}$ in this case are

$$
c_{s 0}^{2}=\frac{P_{0 X}}{\rho_{0 X}}, \quad \Gamma_{0}=0,
$$

and the sound speed agrees with the adiabatic sound speed defined by $\delta P=c_{s}^{2} \delta \rho$. From this fact, it is expected that dynamics of the scalar field system should somehow resemble that of a perfect fluid with equation of state $P=P(\rho)$. This expectation turns out to be essentially correct. In fact, in Appendix we see that these two systems have essentially the same general solutions up to $O\left(\epsilon^{2}\right)$ in the gradient expansion except for the following one difference. The 3 -velocity $u_{i}$ for the scalar field system does not include transverse mode but that for the perfect fluid in general does. The evolution of other quantities such as $\delta, \chi, \psi, \tilde{\gamma}_{i j}$ and $\tilde{A}_{i j}$ are the same.

Note that ghost condensation [44, 45, 46] also has a similar low energy effective Lagrangian but includes terms like $\left(\vec{\nabla}^{2} \pi\right)^{2}$ as well, where $\vec{\nabla}$ is the spatial gradient and $\pi$ is perturbation of the scalar field. Indeed, those additional terms play important roles in generation of primordial density perturbations [32] and infra-red modification of gravity [44]. In other words, as easily seen by doing a proper scaling analysis, a Lagrangian of the form $P(X)$ without those additional terms can not describe ghost condensation in general even at low energies. However, if we are interested in a situation where the the Hubble scale during inflation is longer than the scale of IR modification of gravity then we can safely use the present formalism to investigate classical evolution of cosmological perturbation at superhorizon scales. However, if the scale of IR modification is longer then the present formulation is not valid. In this case we probably need to extend the present formulation to include the higher derivative term. More detailed investigation will be considered in future publication.

\section{B. Canonical scalar}

Next let us consider a canonical scalar field, i.e.

$$
P(X, \phi)=\frac{X}{2}-V(\phi),
$$

where $V(\phi)$ is a potential. In this case, we can obtain

$$
c_{s 0}^{2}=1, \quad \Gamma_{0}=-\frac{2 V_{\phi 0}}{\rho_{0}},
$$

where $V_{\phi 0} \equiv d V\left(\phi_{0}\right) / d \phi_{0}$. The general solution can be read off from (4.16). In particular, $\chi$ is given by

$$
\chi=-\frac{2 \rho_{*} a_{*}^{2}}{3 \dot{\phi}_{0}^{3} a^{3}}\left[\left(2 a \phi_{0}+V_{\phi 0} \int_{t_{0}}^{t} a\left(t^{\prime}\right) d t^{\prime}\right) Q^{(2)}\left(x^{k}\right)+V_{\phi 0} \Pi^{(2)}\left(x^{k}\right)\right]+O\left(\epsilon^{4}\right),
$$

where $\dot{\phi}_{0}=\partial_{t} \phi_{0}$. If we set $Q^{(2)}\left(x^{k}\right) \rho_{*} a_{*}^{2}=-3 \dot{\phi}_{*} C\left(x^{k}\right)$ and $\Pi^{(2)}\left(x^{k}\right) \rho_{*} a_{*}^{2}=-3 \dot{\phi}_{*} D\left(x^{k}\right)$, then this reduces to eq. (C29) of [25]. 


\section{DBI scalar}

We now consider a scalar field described by the so called DBI action. This kind of scalar is considered as an inflaton in an interesting class of inflationary models called DBI inflation [34, 35]. In a simple case the inflaton scalar field corresponds to the radial position of a D3-brane in a warped compactification.

For a warped throat background, the 10-dimensional metric takes the following form

$$
d s^{2}=h^{2}(\rho) \eta_{\mu \nu} d x^{\mu} d x^{\nu}+h^{-2}(\rho)\left(d \rho^{2}+\rho^{2} g_{m n}^{(5)} d x^{m} d x^{n}\right)
$$

where $h(\rho)$ is a warp factor, $x^{\mu}(\mu=0,1,2,3)$ are external 4-dimensional coordinates, $\rho$ is the radial coordinate in the warped throat, and $x^{m}(m=5,6,7,8,9)$ are the internal 5-dimensional angular coordinates. Considering the radial position $\rho$ of a D3-brane in this background as a scalar field in the external 4-dimensional spacetime, its dynamics is described by a Dirac-Born-Infeld (DBI) action plus a Chern-Simons term and additional potential terms. Thus, the Lagrangian $P(X, \phi)$ is

$$
P(X, \phi)=-T(\phi) \sqrt{1-X / T(\phi)}+T(\phi)-V(\phi)
$$

where $\phi \equiv T_{3}^{1 / 2} \rho$ is the scalar field describing the D3-brane position, $T(\phi) \equiv T_{3} h^{4}(\phi)$ is the warped brane tension, and $V(\phi)$ is the inflaton potential. Here, $T_{3}$ is the D3-brane tension. The Lagrangian (5.6) also applies to the case where the inflaton $\phi$ is the radial position of a wrapped D5- or D7-brane [47].

For the Lagrangian (5.6) we obtain

$$
c_{s 0}^{2}=\frac{1}{\gamma^{2}}, \quad \Gamma_{0}=\frac{1}{\rho_{0}}\left[-\frac{2 T_{\phi 0}}{\gamma}+\left(T_{\phi 0}-V_{\phi 0}\right)\left(1+\frac{1}{\gamma^{2}}\right)\right],
$$

where $T_{\phi 0} \equiv d T\left(\phi_{0}\right) / d \phi_{0}$ and $\gamma \equiv 1 / \sqrt{1-X_{0} / T\left(\phi_{0}\right)}$. In a non-relativistic limit $(\gamma \simeq 1)$, $c_{s 0}^{2}$ and $\Gamma_{0}$ reduce to those for the canonical scalar field discussed in subsection $\mathrm{VB}$.

\section{SUMMARY AND DISCUSSION}

We have developed a theory of nonlinear cosmological perturbations on superhorizon scales for a scalar field described by a Lagrangian of the form $P(X, \phi)$, where $X=-\partial^{\mu} \phi \partial_{\mu} \phi$ and $\phi$ is the scalar field, and also for a perfect fluid with a general equation of state $P=P(\rho)$. The general solutions valid up to the order $O\left(\epsilon^{2}\right)$ in the spatial gradient expansion have been presented in subsection IVC for the scalar field system and in Appendix A for the perfect fluid.

This formalism can be applied to many interesting circumstances. Some particular examples have been listed in Sec. V] including a scalar with shift symmetry, a canonical scalar and a DBI scalar. (As shown in Appendix A it can be applied also for a perfect fluid with general equation of state $P=P(\rho)$.) Thus, the formalism can be used to investigate superhorizon evolution of nonlinear cosmological perturbations in k-inflation and DBI inflation. As explained in Sec. I. non-Gaussianity can be affected by order $O\left(\epsilon^{2}\right)$ corrections if, e.g. there is a stage which violates the slow roll conditions.

For ghost inflation, applicability of the present formulation seems a bit subtle as briefly discussed in subsection V A. If the Hubble scale is longer than the scale of IR modification of gravity then we can use the present formalism to investigate superhorizon perturbations. On the other hand, if one is interested in a situation where the scale of IR modification is longer then the present formulation is not valid. We hope to address this issue in more detail in future publication.

Recently, models of multi-field DBI have been also studied to investigate large non-Gaussianity by [22, 23]. Here our formulation has been developed in a single scalar field, however, we also plan to extend it to a system of multi-field scalar in the future.

\section{Acknowledgments}

Y.T. would like to thank Kei-ichi Maeda, Tsutomu Kobayashi, and Shuichiro Yokoyama for their comments and/or discussions on this work, and also wish to show our acknowledgement to the financial support by Waseda University. The work of S.M. was supported in part by MEXT through a Grant-in-Aid for Young Scientists (B) No. 17740134, and by JSPS through a Grant-in-Aid for Creative Scientific Research No. 19GS0219 and through a Grant-in-Aid for Scientific Research (B) No. 19340054. This work was supported by World Premier International Research Center InitiativeWPI Initiative), MEXT, Japan. 


\section{APPENDIX A: PERFECT FLUID WITH $P=P(\rho)$}

In this appendix, we shall consider a universe dominated by a perfect fluid with a general equation of state $P=P(\rho)$. The stress-energy tensor is given by

$$
T_{\mu \nu}=(\rho+P) u_{\mu} u_{\nu}+P g_{\mu \nu}, \quad P=P(\rho) .
$$

The formulation in this case is similar to that presented in Sec. III and Sec. IV for a scalar field. Main differences come from the form of the pressure perturbation $p$.

As in Sec. III and Sec. IV we employ the ADM formalism and the gradient expansion in the uniform Hubble slicing. In the ADM formalism, the dynamical equations are given by (3.9), with the two constraint equations (3.7) and (3.8). We further decompose the spatial metric and the extrinsic curvature as (3.10). Then we obtain the firstorder equations (3.11) and (3.12) for the spatial metric $\left(\psi, \tilde{\gamma}_{i j}\right)$, and (3.13) and (3.14) for the extrinsic curvature $\left(K, \tilde{A}_{i j}\right)$. The Hamiltonian and momentum constraint are, respectively, (3.15) and (3.16). The conservation equation $\nabla_{\mu} T_{\nu}^{\mu}=0$ is given by (3.17) and (3.18). We adopt the uniform Hubble slicing as (3.19).

Next, we will employ the gradient expansion. In this approach we introduce a flat FRW universe $\left(a(t), \rho_{0}(t)\right)$ as a background and suppose that the characteristic length scale $L$ of perturbations is longer than the Hubble length scale $1 / H$ of the background, i.e. $H L \gg 1$. Therefore, we consider $\epsilon \equiv 1 /(H L)$ as a small parameter and systematically expand our equations by $\epsilon$, considering a spatial derivative acted on perturbations is of order $O(\epsilon)$. The background flat FRW universe $\left(a(t), \rho_{0}(t)\right)$ satisfies the Friedmann equation and the conservation equation $\partial_{t} \rho_{0}+3 H\left(\rho_{0}+P_{0}\right)=0$, where $P_{0}=P\left(\rho_{0}\right)$. We can estimate order of magnitude of various quantities by using the assumption (3.22) and (3.23). As a result, we have the estimates shown in (3.32) except that the condition $\pi=O\left(\epsilon^{2}\right)$ does not exist in the present case. Then substituting the order of magnitude (3.32) into the basic equations gives leading order equations as (3.33)- -3.40$)$.

By using these equations and background conservation equation, equations for $\delta$ and $u_{i}$ are easily obtained as (3.41) and (3.42). They are easily solved as (4.6) and (4.7). The traceless part of the extrinsic curvature $\tilde{A}_{i j}$ is solved by using the leading part of $\psi$ and $\tilde{\gamma}_{i j}$, (4.1) and (4.2), as (4.5). The 'constants' of integration are not independent but are related to each other by the two constraint equations as (4.8). However, in the present case there is no relation like (4.11), and then $C_{i}^{(3)}$ contains transverse component as well as the longitudinal component.

For $P=P(\rho)$, perturbation of the pressure $p \equiv P-P_{0}$ is given by

$$
p=\rho_{0} c_{s 0}^{2} \delta+O\left(\epsilon^{4}\right), \quad c_{s 0}^{2}=\frac{d P_{0}}{d \rho_{0}} .
$$

By using (3.40), we obtain the solution for $\chi$ as

$$
\chi=-\frac{\left(1+3 c_{s 0}^{2}\right) R\left[\left(L^{(0)}\right)^{4} f^{(0)}\right]}{6 \kappa^{2}\left(\rho_{0}+P_{0}\right) a^{2}}+O\left(\epsilon^{4}\right) .
$$

In order to obtain a general solution valid up to $O\left(\epsilon^{2}\right)$, we further seek $O\left(\epsilon^{2}\right)$ corrections to $\psi$ and $\tilde{\gamma}_{i j}$. For this purpose we adopt the gauge condition (4.12). With this gauge choice, the $O\left(\epsilon^{2}\right)$ part of $\tilde{\gamma}_{i j}$ can be obtained by using the solution of $\tilde{A}_{i j}$ as (4.14). Similarly, by using the solution of $\chi(\underline{\mathrm{A} 3})$, the $O\left(\epsilon^{2}\right)$ correction to $\psi$ is obtained as (4.15), provided that $\chi$ in the present case is given by (A3).

In summary we have obtained a general solution valid up to $O\left(\epsilon^{2}\right)$ for the perfect fluid with $P=P(\rho)$.

$$
\begin{aligned}
\delta & =\frac{1}{2 \kappa^{2} \rho_{0} a^{2}} R\left[\left(L^{(0)}\right)^{4} f^{(0)}\right]+O\left(\epsilon^{4}\right) \\
u_{i} & =\frac{1}{6 \kappa^{2}\left(\rho_{0}+P_{0}\right) a^{3}}\left(\partial_{i} R\left[\left(L^{(0)}\right)^{4} f^{(0)}\right] \int_{t_{0}}^{t} a\left(t^{\prime}\right) d t^{\prime}+C_{i}^{(3)}\right)+O\left(\epsilon^{5}\right), \\
\chi & =-\frac{1+3 c_{s 0}^{2}}{6 \kappa^{2}\left(\rho_{0}+P_{0}\right) a^{2}} R\left[\left(L^{(0)}\right)^{4} f^{(0)}\right]+O\left(\epsilon^{4}\right), \\
\psi & =L^{(0)}\left(1+\frac{1}{2} \int_{t_{0}}^{t} H\left(t^{\prime}\right) \chi\left(t^{\prime}\right) d t^{\prime}\right)+O\left(\epsilon^{4}\right), \\
\tilde{\gamma}_{i j} & =f_{i j}^{(0)}-2\left(F_{i j}^{(2)} \int_{t_{0}}^{t} \frac{d t^{\prime}}{a^{3}\left(t^{\prime}\right)} \int_{t_{0}}^{t^{\prime}} a\left(t^{\prime \prime}\right) d t^{\prime \prime}+C_{i j}^{(2)} \int_{t_{0}}^{t} \frac{d t^{\prime}}{a^{3}\left(t^{\prime}\right)}\right)+O\left(\epsilon^{4}\right), \\
\tilde{A}_{i j} & =\frac{1}{a^{3}}\left(F_{i j}^{(2)} \int_{t_{0}}^{t} a\left(t^{\prime}\right) d t^{\prime}+C_{i j}^{(2)}\right)+O\left(\epsilon^{4}\right),
\end{aligned}
$$


where

$$
C_{i}^{(3)}=\frac{3}{\left(L^{(0)}\right)^{6} \kappa^{2} \rho_{*} a_{*}^{2}} f_{(0)}^{j k} \tilde{D}_{j}^{(0)}\left[\left(L^{(0)}\right)^{6} C_{k i}^{(2)}\right]+O\left(\epsilon^{5}\right) .
$$

Here $F_{i j}^{(2)}$ is defined by (4.4), and 'constants' of integration $L^{(0)}, f_{i j}^{(0)}$ and $C_{i j}^{(2)}$ depend only on the spatial coordinates $\left\{x^{k}\right\}(k=1,2,3)$ and satisfy

$$
\begin{gathered}
f_{i j}^{(0)}=f_{j i}^{(0)}, \quad \operatorname{det}\left(f_{i j}^{(0)}\right)=1, \\
C_{i j}^{(2)}=C_{j i}^{(2)}, \quad f_{(0)}^{i j} C_{i j}^{(2)}=0 .
\end{gathered}
$$

Here, $f_{(0)}^{i j}$ is the inverse matrix of $f_{i j}^{(0)}$ and $\tilde{D}^{(0)}$ is the covariant derivative compatible with $f_{i j}^{(0)}$. Compared with the solution (4.16) for the scalar field system, only differences are: (i) $\pi$ does not exist; (ii) $\Gamma_{0}=0$; and (iii) $C_{i}^{(3)}$ includes not only a longitudinal component but also transverse components.

The number of degrees of freedom included in each 'constant' of integration is

$$
\begin{array}{lll}
L^{(0)} & \cdots & 1 \text { growing adiabatic mode of density perturbation }=1 \text { component } \\
f_{i j}^{(0)} & \cdots & 2 \text { tensor growing modes }=5 \text { components }-3 \text { gauge }, \\
C_{i j}^{(2)} & \cdots & 2 \text { tensor decaying modes }+3 \text { velocity }=5 \text { components } .
\end{array}
$$

[1] G. F. Smoot et al., "Structure in the COBE differential microwave radiometer first year maps," Astrophys. J. 396, L1 (1992).

C. L. Bennett et al., "4-Year COBE DMR Cosmic Microwave Background Observations: Maps and Basic Results," Astrophys. J. 464, L1 (1996) arXiv:astro-ph/9601067.

[2] J. M. Bardeen, "Gauge Invariant Cosmological Perturbations," Phys. Rev. D 22 (1980) 1882.

[3] H. Kodama and M. Sasaki, "Cosmological Perturbation Theory," Prog. Theor. Phys. Suppl. 78 (1984) 1.

[4] V. F. Mukhanov, H. A. Feldman and R. H. Brandenberger, "Theory of cosmological perturbations. Part 1. Classical perturbations. Part 2. Quantum theory of perturbations. Part 3. Extensions," Phys. Rept. 215, 203 (1992).

[5] E. Komatsu et al. [WMAP Collaboration], "First Year Wilkinson Microwave Anisotropy Probe (WMAP) Observations: Tests of Gaussianity," Astrophys. J. Suppl. 148, 119 (2003) arXiv:astro-ph/0302223.

D. N. Spergel et al. [WMAP Collaboration], "Wilkinson Microwave Anisotropy Probe (WMAP) three year results: Implications for cosmology," Astrophys. J. Suppl. 170, 377 (2007) arXiv:astro-ph/0603449.

[6] E. Komatsu et al. [WMAP Collaboration], "Five-Year Wilkinson Microwave Anisotropy Probe Observations:Cosmological Interpretation," arXiv:0803.0547 [astro-ph].

[7] [Planck Collaboration], "Planck: The scientific programme," arXiv:astro-ph/0604069

[8] N. Bartolo, E. Komatsu, S. Matarrese and A. Riotto, "Non-Gaussianity from inflation: Theory and observations," Phys. Rept. 402, 103 (2004) arXiv:astro-ph/0406398.

[9] J. M. Maldacena, "Non-Gaussian features of primordial fluctuations in single field inflationary models," JHEP 0305, 013 (2003) arXiv:astro-ph/0210603.

[10] D. Seery and J. E. Lidsey, "Primordial non-gaussianities in single field inflation," JCAP 0506, 003 (2005) arXiv:astro-ph/0503692.

[11] K. A. Malik and D. Wands, "Evolution of second order cosmological perturbations," Class. Quant. Grav. 21, L65 (2004) arXiv:astro-ph/0307055.

[12] G. I. Rigopoulos and E. P. S. Shellard, "The separate universe approach and the evolution of nonlinear superhorizon cosmological perturbations," Phys. Rev. D 68, 123518 (2003) arXiv:astro-ph/0306620.

G. I. Rigopoulos and E. P. S. Shellard, "Non-linear inflationary perturbations," JCAP 0510, 006 (2005) arXiv:astro-ph/0405185.

[13] D. H. Lyth, K. A. Malik and M. Sasaki, "A general proof of the conservation of the curvature perturbation," JCAP 0505, 004 (2005) arXiv:astro-ph/0411220.

[14] D. Langlois and F. Vernizzi, "Evolution of non-linear cosmological perturbations," Phys. Rev. Lett. 95, 091303 (2005) arXiv:astro-ph/0503416. D. Langlois and F. Vernizzi, "Nonlinear perturbations of cosmological scalar fields," JCAP 0702, 017 (2007) arXiv:astro-ph/0610064.

[15] D. H. Lyth and Y. Rodriguez, "The inflationary prediction for primordial non-gaussianity," Phys. Rev. Lett. 95, 121302 (2005) arXiv:astro-ph/0504045. 
[16] E. Komatsu and D. N. Spergel, "Acoustic signatures in the primary microwave background bispectrum," Phys. Rev. D 63, 063002 (2001) arXiv:astro-ph/0005036.

[17] F. Vernizzi and D. Wands, "Non-Gaussianities in two-field inflation," JCAP 0605, 019 (2006) arXiv:astro-ph/0603799.

[18] G. I. Rigopoulos, E. P. S. Shellard and B. J. W. van Tent, "Non-linear perturbations in multiple-field inflation," Phys. Rev. D 73, 083521 (2006) arXiv:astro-ph/0504508.

[19] T. Battefeld and R. Easther, "Non-gaussianities in multi-field inflation," JCAP 0703, 020 (2007) arXiv:astro-ph/0610296.

[20] S. Yokoyama, T. Suyama and T. Tanaka, "Primordial Non-Gaussianity in Multi-Scalar Slow-Roll Inflation," JCAP 0707, 013 (2007) arXiv:0705.3178 [astro-ph]].

S. Yokoyama, T. Suyama and T. Tanaka, "Primordial Non-Gaussianity in Multi-Scalar Inflation," Phys. Rev. D 77, 083511 (2008) arXiv:0711.2920 [astro-ph]].

[21] M. Sasaki, "Multi-brid inflation and non-Gaussianity," Prog. Theor. Phys. 120, 159 (2008) arXiv:0805.0974 [astro-ph]]. A. Naruko and M. Sasaki, "Large non-Gaussianity from multi-brid inflation," arXiv:0807.0180 [astro-ph].

[22] F. Arroja, S. Mizuno and K. Koyama, "Non-gaussianity from the bispectrum in general multiple field inflation," JCAP 0808, 015 (2008) arXiv:0806.0619 [astro-ph]].

[23] D. Langlois, S. Renaux-Petel, D. A. Steer and T. Tanaka, "Primordial perturbations and non-Gaussianities in DBI and general multi-field inflation," arXiv:0806.0336 [hep-th].

D. Langlois, S. Renaux-Petel, D. A. Steer and T. Tanaka, "Primordial fluctuations and non-Gaussianities in multi-field DBI inflation," Phys. Rev. Lett. 101, 061301 (2008) arXiv:0804.3139 [hep-th]].

[24] Y. Tanaka and M. Sasaki, "Gradient expansion approach to nonlinear superhorizon perturbations," Prog. Theor. Phys. 117, 633 (2007) arXiv:gr-qc/0612191.

[25] Y. Tanaka and M. Sasaki, "Gradient expansion approach to nonlinear superhorizon perturbations II - a single scalar field -," Prog. Theor. Phys. 118, 455 (2007) arXiv:0706.0678 [gr-qc]].

[26] T. Moroi and T. Takahashi, "Effects of cosmological moduli fields on cosmic microwave background," Phys. Lett. B 522, 215 (2001) [Erratum-ibid. B 539, 303 (2002)] arXiv:hep-ph/0110096.

[27] D. H. Lyth and D. Wands, "Generating the curvature perturbation without an inflaton," Phys. Lett. B 524, 5 (2002) arXiv:hep-ph/0110002.

[28] K. A. Malik and D. H. Lyth, "A numerical study of non-gaussianity in the curvaton scenario," JCAP 0609,008 (2006) arXiv:astro-ph/0604387.

[29] M. Sasaki, J. Valiviita and D. Wands, "Non-gaussianity of the primordial perturbation in the curvaton model," Phys. Rev. D 74, 103003 (2006) arXiv:astro-ph/0607627.

[30] J. Garriga and V. F. Mukhanov, "Perturbations in k-inflation," Phys. Lett. B 458, 219 (1999) arXiv:hep-th/9904176].

[31] C. Armendariz-Picon, T. Damour and V. F. Mukhanov, "k-inflation," Phys. Lett. B 458, 209 (1999) arXiv:hep-th/9904075.

[32] N. Arkani-Hamed, P. Creminelli, S. Mukohyama and M. Zaldarriaga, "Ghost inflation," JCAP 0404, 001 (2004) arXiv:hep-th/0312100.

[33] L. Senatore, "Tilted ghost inflation," Phys. Rev. D 71, 043512 (2005) arXiv:astro-ph/0406187.

[34] E. Silverstein and D. Tong, "Scalar speed limits and cosmology: Acceleration from D-cceleration," Phys. Rev. D 70, 103505 (2004) arXiv:hep-th/0310221.

[35] M. Alishahiha, E. Silverstein and D. Tong, "DBI in the sky," Phys. Rev. D 70, 123505 (2004) arXiv:hep-th/0404084.

[36] S. Weinberg, "Effective Field Theory for Inflation," Phys. Rev. D 77, 123541 (2008) arXiv:0804.4291 [hep-th]].

[37] X. Chen, M. x. Huang, S. Kachru and G. Shiu, "Observational signatures and non-Gaussianities of general single field inflation," JCAP 0701, 002 (2007) arXiv:hep-th/0605045.

[38] V. Acquaviva, N. Bartolo, S. Matarrese and A. Riotto, "Second-order cosmological perturbations from inflation," Nucl. Phys. B 667, 119 (2003) arXiv:astro-ph/0209156.

[39] D. S. Salopek and J. R. Bond, "Nonlinear evolution of long wavelength metric fluctuations in inflationary models," Phys. Rev. D 42, 3936 (1990).

[40] M. Sasaki and T. Tanaka, "Super-horizon scale dynamics of multi-scalar inflation," Prog. Theor. Phys. 99, 763 (1998) arXiv:gr-qc/9801017.

[41] D. Wands, K. A. Malik, D. H. Lyth and A. R. Liddle, "A new approach to the evolution of cosmological perturbations on large scales," Phys. Rev. D 62, 043527 (2000) arXiv:astro-ph/0003278.

[42] S. M. Leach, M. Sasaki, D. Wands and A. R. Liddle, "Enhancement of superhorizon scale inflationary curvature perturbations," Phys. Rev. D 64, 023512 (2001) arXiv:astro-ph/0101406.

[43] R. K. Jain, P. Chingangbam and L. Sriramkumar, "Amplification of tachyonic perturbations at super-Hubble scales," JCAP 0710, 003 (2007) arXiv:astro-ph/0703762.

[44] N. Arkani-Hamed, H. C. Cheng, M. A. Luty and S. Mukohyama, "Ghost condensation and a consistent infrared modification of gravity," JHEP 0405, 074 (2004) arXiv:hep-th/0312099.

[45] N. Arkani-Hamed, H. C. Cheng, M. A. Luty, S. Mukohyama and T. Wiseman, "Dynamics of gravity in a Higgs phase," JHEP 0701, 036 (2007) arXiv:hep-ph/0507120.

[46] S. Mukohyama, "Accelerating universe and cosmological perturbation in the ghost condensate," JCAP 0610, 011 (2006) arXiv:hep-th/0607181.

[47] T. Kobayashi, S. Mukohyama and S. Kinoshita, "Constraints on Wrapped DBI Inflation in a Warped Throat," JCAP 0801, 028 (2008) arXiv:0708.4285 [hep-th]]. 